\title{
BLOW-UP PROBLEM IN NONLINEAR PARABOLIC EQUATIONS
}

\author{
NURUMOVA A.YU \\ National University of Uzbekistan, Tashkent, Uzbekistan
}

\begin{abstract}
The aim of the paper is to present an introduction to the subject of the formation of singularity in nonlinear evolution problems, usually called an explosion. In short, we are interested in a situation where, starting from a smooth initial configuration and after the first period of classical evolution, the solution (or in some cases its derivatives) becomes infinite in a finite time due to the cumulative effect of nonlinearities. We concentrate on problems related to differential equations of parabolic type, or on systems of such equations.

Two new methods for the numerical integration of Cauchy problems for ODEs with inflatable solutions described. The first method based on the application of a differential transformation, where the first derivative (defined in the original equation) is selected as a new independent variable. The second method is based on the introduction of a new nonlocal variable that reduces $O D E$ to a system of related ODEs. Both methods lead to problems whose solutions do not have explosive singular points; therefore, standard numerical methods can be applied. The effectiveness of the proposed methods is illustrated by several test tasks.
\end{abstract}

KEYWORDS: Nonlinear differential equations, Blowing solutions, Numerical methods, Differential transformations, Nonlocal transformations\& Test problems

Received: May 16, 2020; Accepted: Jun 06, 2020; Published: Jun 30, 2020; Paper Id.: IJMPERDJUN2020330

\section{INTRODUCTION}

A large number of processes of the applied Sciences can be simulated using the evolution equations involving deferential operators, or systems of such equations. To obtain well-defined solutions of the equation supplemented by suitable additional conditions (usually initial and boundary conditions). Standard differential theories involve linear operators, and they developed an extensive theory. Nonlinear partial differential equations and systems have a number of properties, which are absent in the linear theories; these nonlinear properties are often associated with important features of real world phenomena which the mathematical model needs to describe; at the same time these new properties are closely connected with essential new difficulties of mathematical treatment. The study of nonlinear processes is a constant source of new challenges and motivates the introduction of new methods in the areas of mathematical analysis, partial differential equations and other disciplines, becoming a most active area of mathematical research in the latter part of this century. The success of the new methods of modern analysis has enabled. Mathematicsgive rigorous answers to important questions of the nonlinear world. One of the most remarkable properties that distinguish nonlinear evolution problems from the linear, is the possibility of the appearance of features, starting from perfectly smooth data, but rather with classes of data can be established the theory of existence, uniqueness and continuous dependence. Forminor intervals, the so-called correctness in the small. Although features can arise in linear problems, this happens through the specifics contained in the coefficients or data or problem (fixed features). On the contrary, in nonlinear systems, they may arise from the 
nonlinear mechanisms of the problem, and their time and location determined by mathematical analysis (moving features).

\section{Statement of the Task}

Blow-up and ordinary differential equations. The simplest form of spontaneous singularities in nonlinear problems arises when the variable or variables tend to infinity when time approaches some finite limit $\mathrm{T}>0$. This is what we call the phenomenon of the blow-up. A gap occurs in an elementary (but quite representative) form in the theory of ordinary differential equations (odes), and the simplest example is the initial problem for a real scalar variable $u=u(t)$.

$$
u_{t}=u^{2}, t>0 ; \quad u(0)=a .
$$

for data a $>0$ it is immediate that a unique solution exists in the time interval $0<\mathrm{t}<\mathrm{T}=1 / \mathrm{a}$. Since it is given by the formula $\mathrm{u}(\mathrm{t})=1 /(\mathrm{T}-\mathrm{t})$, one sees that it is a smooth function for $\mathrm{t}<\mathrm{T}$ and also that $\mathrm{u}(\mathrm{t}) \rightarrow \infty$ as $\mathrm{t} \rightarrow \mathrm{T}-$ (limit from the left). We say that the solution blows up at $\mathrm{t}=\mathrm{T}$ and also that $\mathrm{u}(\mathrm{t})$ has blow-up at that time. Blow-up is referred to in Latin languages as blow-up, and in fact the mathematical problems involved aim in many cases at (partially) describing explosive phenomena. Starting from this example, the concept of blow-up can be widely generalized as the phenomenon whereby solutions cease to exist globally in time because of infinite growth of the variables describing the evolution process. Thus, a first step is given by ODE's of the form $u_{t}=u^{p}$, with $p>1$ and, more generally

$$
u_{t}=f(u) \text {, }
$$

where $f$ is positive and, say, continuous, under the condition.

This Osgood's condition in the ODE theory established in 1898 [142] (see [110], Part I.3, and also [168], p. 140) is necessary and sufficient for the occurrence of blow-up in finite time for any solution with positive initial data. More generally, we can think of systems $u t=f(t, u)$ for a vector variable $u \in R n$. In this case we may have blow-up due to the same mechanism if $\mathrm{f}$ is super-linear with respect to $\mathrm{u}$ for $|\mathrm{u}|$ large, and also blow-up due to the singular character of $\mathrm{f}$ with respect to $t$ at certain given times. It is the generalization of the first form that will of concern in these notes. The study of ODE's supplies basic tools and intuitions for the whole theory of blow-up, and, more generally, the study of singularities.

Blow-up in the reaction-diffusion equations: the main problems and a bit of history. The previous questions acquire mathematical complexity and are also of interest for use in various sciences, when the problem has a spatial structure, so that the unknowns depend not only on time, but also on the spatial variable, $u=u(x, t), c x \in \Omega$, domain in $R$ $\mathrm{n}$, while time stretches to the interval $0 \leq \mathrm{t}<\mathrm{T}$. Prominent in the study of inflation problems are the reaction-diffusion equations, cf. [155]. In theories of thermal propagation and combustion, it is natural to consider quasilinear equations of the form:

$$
u_{t}=\nabla \cdot \mathcal{A}(u, \nabla u, x, t)+B(u, \nabla u, x, t)
$$

with standard ellipticity conditions on the operator A and the conditions of growth and regularity as in A and B. Typically, we consider (1.4) as a nonlinear model of heat propagation in a reactive environment, and then the u temperature. It is clear that as a model of the evolutionary process and compared with equation (1.2) this equation introduces a new feature of accounting for the spatial structure of the solutions. The concept of the blow-up is now 
formulated in its simplest form in the following framework.

(i)we start with the correctness of a mathematical problem in a certain framework and for small times; thus, assuming nice regularity conditions on $\mathrm{A}$ and $\mathrm{B}$, we will have a theory of existence and uniqueness, for example, for the Cauchy problem or one of the initial-boundary value problems in a certain class of bounded and nonnegative data, so that the decision to develop, being restricted for some time $0<\mathrm{t}<\mathrm{T}$. (ii) we also Have the theory of regularity and continue in this structure, which says that bounded solutions have the necessary smoothness, so that they can be continued locally in time (see the well-known result in [156]). For classical solutions of parabolic equations this theory is based on the estimates of Schauder. Corresponding estimates exist for weak solutions of equations in divergence form in Sobolev spaces or for fully nonlinear equations, as described in the lectures of Professor Cabra in this volume. Then the blow-up occurs, if the solution becomes innite at some (or many) points when $t$ approaches a certain finite time $\mathrm{T}$. namely, there exists a time $\mathrm{T}<\infty$, called the time of inflating so that the solution is well defined for all $0<\mathrm{t}<\mathrm{T}$, while

$$
\sup _{x \in \Omega}|u(x, t)| \rightarrow \infty \text { as } t \rightarrow T^{-}
$$

with standard ellipticity conditions on the operator A and the conditions of growth and regularity as in A and B. Typically, we consider (1.4) as a nonlinear model of heat propagation in a reactive environment, and then the u temperature. It is clear that as a model of the evolutionary process and compared with equation (1.2) this equation introduces a new feature of accounting for the spatial structure of the solutions. The concept of the blow-up is now formulated in its simplest form in the following framework. (i) we Start with the correctness of a mathematical problem in a certain framework and for small times; thus, assuming nice regularity conditions on A and B, we will have a theory of existence and uniqueness, for example, for the Cauchy problem or one of the initial-boundary value problems in a certain class of bounded and nonnegative data, so that the decision to develop, being restricted for some time $0<\mathrm{t}<\mathrm{T}$. (ii) we also Have the theory of regularity and continue in this structure, which says that bounded solutions have the necessary smoothness, so that they can be continued locally in time (see the well-known result in [156]). For classical solutions of parabolic equations this theory is based on the estimates of Schauder. Corresponding estimates exist for weak solutions of equations in divergence form in Sobolev spaces or for fully nonlinear equations, as described in the lectures of Professor Cabra in this volume. Then the blow-up occurs, if the solution becomes innite at some (or many) points when t approaches a certain finite time $\mathrm{T}$. namely, there exists a time $\mathrm{T}<\infty$, called the time of inflating so that the solution is well defined for all $0<\mathrm{t}<\mathrm{T}$, while

$$
u_{t}=\Delta u+\lambda e^{u}, \quad \lambda>0
$$

what is important in the theory of combustion 1] called the solid fuel model (Frank-Kamenetsky equation), as well as in other fields, cf. [2], [3], [6], [10].

It is also of interest for differential geometry [11] and other applications. The occurrence and type of blow-up depends on the parameter $\lambda$ source data and domain. Another classic blow-up equation

$$
u_{t}=\Delta u+u^{p}
$$

Both of semilinear equations was studied in pioneering works of Fujita. For exponents $p>1$, we have a tendency 
of inflating in (1.7); depending on the value of $\mathrm{p}$ it may happen not only for some but for all solutions of this class. There are a number of other popular models of evolution problems involving nonlinear parabolic equations, possibly degenerate. With a porous medium and operators of $\mathrm{p}$-Laplace

$$
u_{t}=\Delta u^{m}+u^{p}(m>0) \text { and } \quad u_{t}=\nabla \cdot\left(|\nabla u|^{\sigma} \nabla u\right)+u^{p} \quad(\sigma>-1)
$$

results of the first gap was proved in [10] and [12], respectively. Results of local solvability and regularity for the total of these and other degenerate quasilinear equations can be found in the review article [6]. All these models take the form

$$
u_{t}=\mathbf{A}(u)+f(u)
$$

where $\mathrm{A}$ is an elliptic operator of second order, maybe nonlinear and degenerate, representing a diffusion, and $\mathrm{f}(\mathrm{u})$ superlinear function of $\mathrm{u}$ representing reaction. There are some good texts which display many of the results which are known and contain extended lists of references such as books Biberna and Eberly [12], Samara, etc. [11] and Volpert and Khudyaev [10]. However, this is a very active area where there are many new developments. In this sense, there are also some related results of the blow-up in different periods, see, E.g., [13], [15] (both include other nonlinear partial differential equations) and [12] (which consider the semilinear heat equation and numerical methods; this whole issue of J. Comput. Appl. Math. Devoted to various aspects of ventilation, including the numerical results on some open mathematical problems). We intend to Supplement the basic literature, discussed some of the recent events.

Basic questions. Next, we will focus on the analysis of the main issues that arise in the study of the bulge for equations of reaction-diffusion. This list can be suitably adapted to other problems of formation of the singularity. For example, according to [17], the main list includes questions about when, where and how. We offer here an expanded list of six items: (1)whether the blow-up? (2) When? (3) where? (4) How? (5) What will happen then? (6) How to compute it numerically.

Describe what is meant by these questions in brief. For definiteness, we mean the problem of reaction-diffusion, similar to those which were proposed in the last paragraphs, supplied in a spatial region $\Omega \in \mathrm{R} \wedge \mathrm{N}$, but all the concepts can be easily adapted to a more abstract setting.

(1)First question: What is blow-up? The problem of blow-upproperly formulated only when you have selected a suitable class of solutions. Usually the existence and uniqueness of solutions to the problem can be formulated in different functional conditions, and have a blast - this is the inability to continue the solutions in this structure, before or after a certain time. By default, we are dealing with classical solutions, but weak, viscous or other types of generalized solutions may be more natural for this problem. We can consider the cases when the blow-up is fully functional frame and not in another, for example, for classical solutions, but not for weak solutions of L1. This seemingly strange situation does happen in practice, Ms. [11], and only reflects the rich structure of the problems of the blow-up. The General question can be divided into these two aspects:

(1.i)What are the equations and objectives are blow-up in finite time? Form of equation (from the standpoint of its coefficients or, more generally, its structural conditions) and form of the data determine the answer. In the case that the blow-up caused by the reaction term, the condition of superlinearity, as in the analysis of the ODE, but we can find examples of the problem with a spatial structure different from the problem of an ODE. 
(1.ii) If the previous question has a positive answer, we can ask what solutions may explode in finite time? The possibility of the last question is twofold:

Failure occurs for all solutions in the given class or only for some solutions (which should be identified). If the joint solution will not explode, it will be live worldwide in time. Problem for which all solutions blow up, is called the Fujita problem. A classic example is the semilinear heat equation $u t=\_u+u p$, set in a bounded domain with zero Dirichlet conditions, where all classical non-negative solutions explode in a finite time, when the exponent lies in the range p 2 (1, (n $+2) / n$ ). The upper bound is the so-called Fujita indicator.

(2) Second question: when? Given that the blow-up occurs in a finite time, can we estimate the time of the blowup? Indeed, the inflation property can also occur in a less striking form at infinite time, when a solution exists in a given functional structure for all $0<\mathrm{t}<1$, but becomes unlimited for $\mathrm{t}$ ! 1 . Thus, we have an alternative: an blow-up with finite and infinite duration. Indeed, for solutions of reaction-diffusion systems, a table of four options occurs:

$>$ (2.i) global solutions which remain uniformly bounded in time (i.e., no blow-up),

$>$ (2.ii) global solutions with blow up at infinity, infinite-time blow-up,

$>$ (2.iii) solutions with finite-time blow-up (the standard blow-up case), and

$>$ (2.iv) instantaneous blow-up, i.e., the solution blows up at $\mathrm{t}=0$ in a sense to bespecified.

The latter is a very striking nonlinear phenomenon, but we have shown that it occurs for such a simple equation as the exponential reaction equation.

Typical alternatives when $\Omega=$ Rn are: single-point blow-up, where B (u0) consists of a single point (or of a finite number of points), regional blow-up, where the measure of B (u0) is finite and positive, and global blow-up, where $\mathrm{B}(\mathrm{u} 0)$ $=\mathrm{Rn}$.

These notions are naturally adapted when is not the whole Euclidean space. In the Russian literature of the 70-80s these types of blow-up are called LS-regime, S-regime and HS-regime of blow-up, respectively [11]. In the first two cases the blow-up solutions are called localized.

4) the Next question is: how is the blow-up? There are two aspects:

$>$ (4.i) to calculate the rate at which $u$ diverges when approaching $t$ to the time of the inflation and $x$ to the point of blowing, and

$>$ (4.ii) to calculate the profiles of the airflow in a finite time as limits of $u(x, t)$ when $t$ ! $T$ - points the lack of blowing.

Usually the old information is replaced by some norm estimate of $u(\cdot, t)$ when $t$ is approaching to an blow-up, or, better, an asymptotic expansion. In General, the question is how? there is a change of variables (renormalization), which modifies the evolutionary orbit to the limited size followed by a study of the limits of these orbits, which are now not limited to points that are not blown, but rather a set of blown. This usually leads to a common form a stable structure of the blow-up. General classification of the singularity implies the further study of other unstable patterns. As a rule, a singularity a finite time, such as an blow-up, generate a countable discrete (not continuous!) Spectra of structurally different structures are called eigenfunctions of the nonlinear media [14], [15]. This is not the case with infinite time singularities. 
An interesting question concerns the speed of blowing at the approach of t to T. For many equations such as (1.7), scale invariance implies the existence of solutions that are inflated with speed capacity, [16], [18]. Self-similar blast becomes then the usual form and records the speed of the blow-up. However, it was found that for large values of the index $\mathrm{p}$ in (1.7), the actual blowing speed can be faster than self-similar power, a phenomenon called rapid injection. It should be noted that these patterns are unstable. A quick blow-up was detected in a number of problems, and its speed and profiles are difficult to obtain.

(5) the Question, which has until recently received less attention, but it is of great importance for the practical application of mathematical models related to the blow-up is: What happens after the singularity of a rupture in finite time? This is a problemcontinue after the blow-up, is referred to as the blow-up. The main technical premise is to find a suitable permanent solution. This is done in our study, see Sections 6-7, by means of monotone approximation of the reaction member and data so that the approximate problems have global solutions.

Taking the limit, we can decide whether the solution is trivial (i.e., identically infinite) after T or not [13]. This is a natural approach in the application to thermal propagation and combustion. With this method, in essence, there are three alternatives:

(5i) the Solution cannot be continued.In the models that we discuss below, this is because, if you continue, it should be infinite everywhere in the natural sense. We call it Full blast.

$>$ (5ii) The solution can be continued in a certain space-time region after $\mathrm{T}$, but it is infinite in complement, incomplete blow-up.

> (5iii) The solution again becomes limited after T. This is a temporary blow-up. We found it in the form of an blow-up peak, where it becomes limited immediately after T. In the models studied so far, this is a very unstable phenomenon, a transition between more stable evolutionary patterns.

\section{Examples of Test Problems and Numerical Solutions}

Example 1. Consider the model Cauchy problem for the first-order ODE

$$
y_{x}^{\prime}=y^{2} \quad(x>0) ; \quad y(0)=a,
$$

where $a>0$. The exact solution of this problem has the form

$$
y_{x x}^{\prime \prime}=f\left(x, y, y_{x}^{\prime}\right) \quad\left(x>x_{0}\right) ; \quad y\left(x_{0}\right)=y_{0}, \quad y_{x}^{\prime}\left(x_{0}\right)=y_{1}
$$

First, we present the ODE (1) as a system of equations

$$
y_{x}^{\prime}=t, \quad y_{x x}^{\prime \prime}=f(x, y, t)
$$

wherefx and fy are the respective partial derivatives of $\mathrm{f}$. Eliminating first dy, and then dx from (3), we obtain a system of the first-order coupled equations 


$$
x_{t}^{\prime}=\frac{1}{f_{x}+t f_{y}}, \quad y_{t}^{\prime}=\frac{t}{f_{x}+t f_{y}} \quad\left(t>t_{0}\right) \text {, }
$$

which must be supplemented by the initial conditions

$$
x\left(t_{0}\right)=x_{0}, \quad y\left(t_{0}\right)=y_{0}, \quad t_{0}=f\left(x_{0}, y_{0}\right)
$$

The exact solution of this problem has the form

$$
x=\frac{1}{a}-\frac{1}{\sqrt{t}}, \quad y=\sqrt{t} \quad\left(t \geq a^{2}\right) .
$$

$\mathrm{t}$ has no features; the function $\mathrm{x}=\mathrm{x}(\mathrm{t})$ monotonically increases for $\mathrm{t}>\mathrm{a} 2$, tending to the desired limit value $\mathrm{x} *=$ $\lim \mathrm{t} \rightarrow \infty \mathrm{x}(\mathrm{t})=1 / \mathrm{a}$, and the function $\mathrm{y}=\mathrm{y}(\mathrm{t})$ monotonously increases with increasing $\mathrm{m}$. Solution (9) for system (11) is a solution to the original problem (6) in a parametric form.

Let $\mathrm{a}=1$. Figure 1a shows a comparison of the exact solution (10) of the Cauchy problem for one equation (6) with the numerical solution of the system of equations (11) obtained by the classical Runge - Kutta method (with step size $=0.2)$
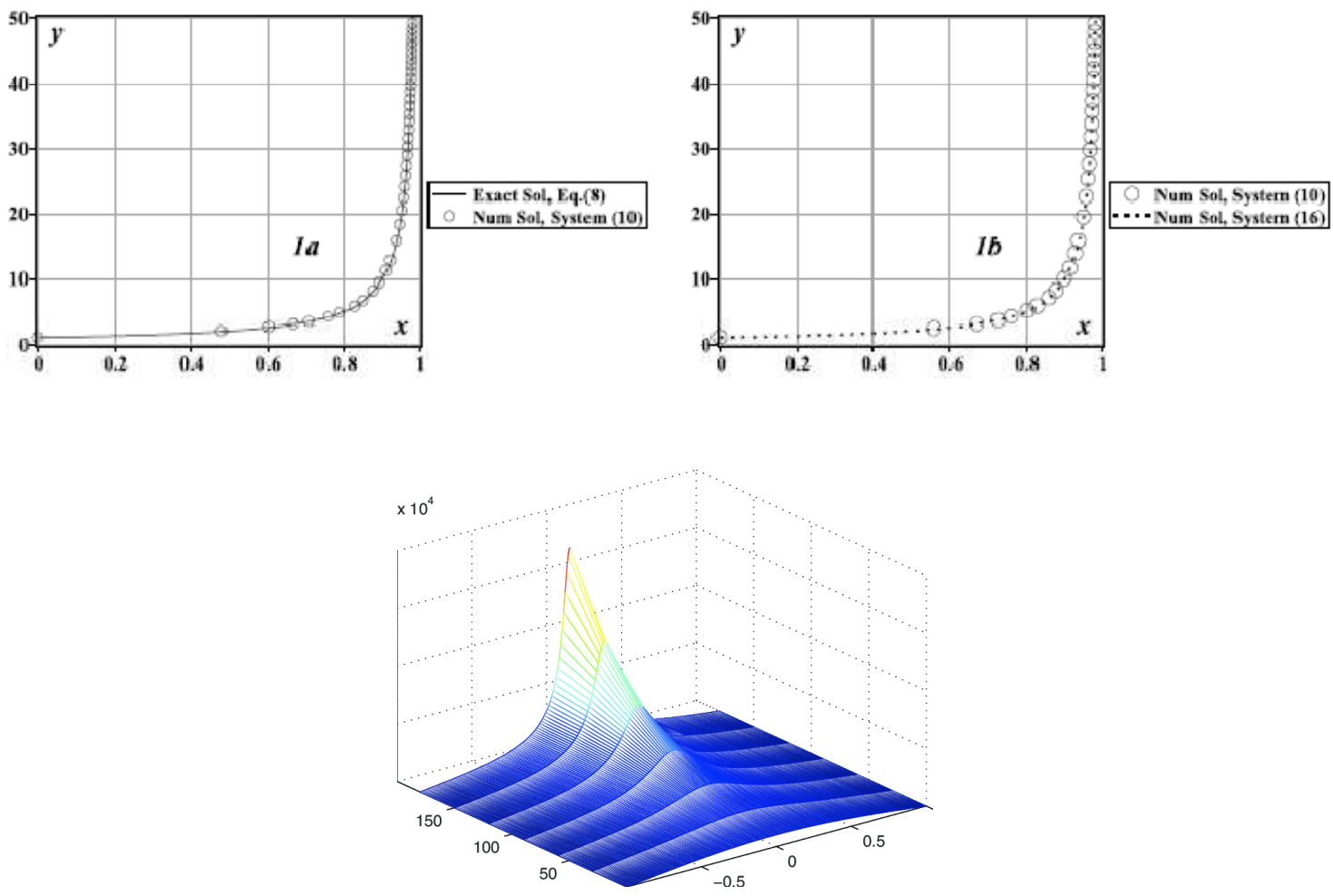

Figure 1: 1(a): Exact Solution of the Cauchy Problem and Numerical Solution of System ; 1(b): numerical solutions of $\operatorname{systems}((a=1$ and $x *=1)$. 


\section{Problems for Second-Order Equations}

Solution method based on a differential transformation The Cauchy problem for the second-order differential equation has the form

$$
y_{x x}^{\prime \prime}=f\left(x, y, y_{x}^{\prime}\right) \quad\left(x>x_{0}\right) ; \quad y\left(x_{0}\right)=y_{0}, \quad y_{x}^{\prime}\left(x_{0}\right)=y_{1}
$$

Note that the exact solutions of equations of the form (15), which can be used for test problems with blow-up solutions, can be found, for example, in $[7,8]$.

First, we represent ODE (15) as an equivalent system of equation

$$
y_{x}^{\prime}=t, \quad y_{x x}^{\prime \prime}=f(x, y, t)
$$

where $\mathrm{y}=\mathrm{y}(\mathrm{x})$ and $\mathrm{t}=\mathrm{t}(\mathrm{x})$ are unknown functions. Taking into account (16), we derive further a standard system of equations, assuming that $\mathrm{y}=\mathrm{y}(\mathrm{t})$ and $\mathrm{x}=\mathrm{x}(\mathrm{t})$. To do this, we differentiate the first equation in (16) with respect to $\mathrm{t}$. We have $\left(y^{\prime} x\right)^{\prime} t=1$. Taking into account the relations $y^{\prime} t=t x^{\prime} t$ (follows from the first equation of $\left.(16)\right)$ and $\left(y^{\prime} x\right){ }^{\prime} t=y^{\prime \prime}$ $\mathrm{xx} / \mathrm{t}^{\prime} \mathrm{x}=\mathrm{x}^{\prime} \mathrm{t} \mathrm{y}^{\prime \prime} \mathrm{xx}$, we get further

$$
x_{t}^{\prime} y_{x x}^{\prime \prime}=1
$$

If we eliminate the second derivative $\mathrm{y}$ " $\mathrm{xx}$ by using a second equation of (16), we obtain the first-order equation

$$
x_{t}^{\prime}=\frac{1}{f(x, y, t)} \text {. }
$$
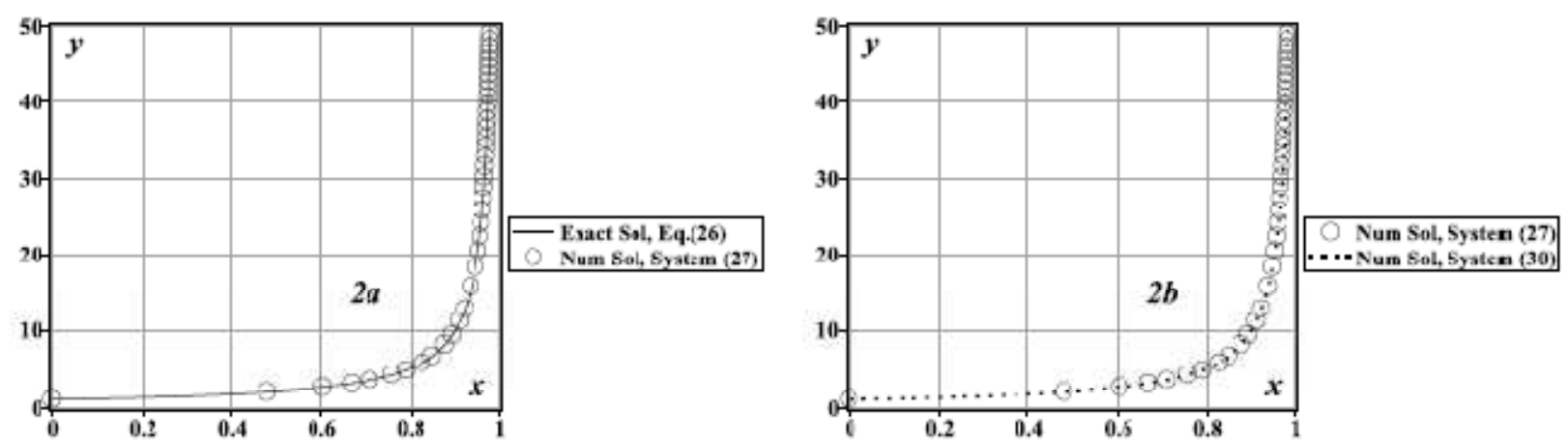


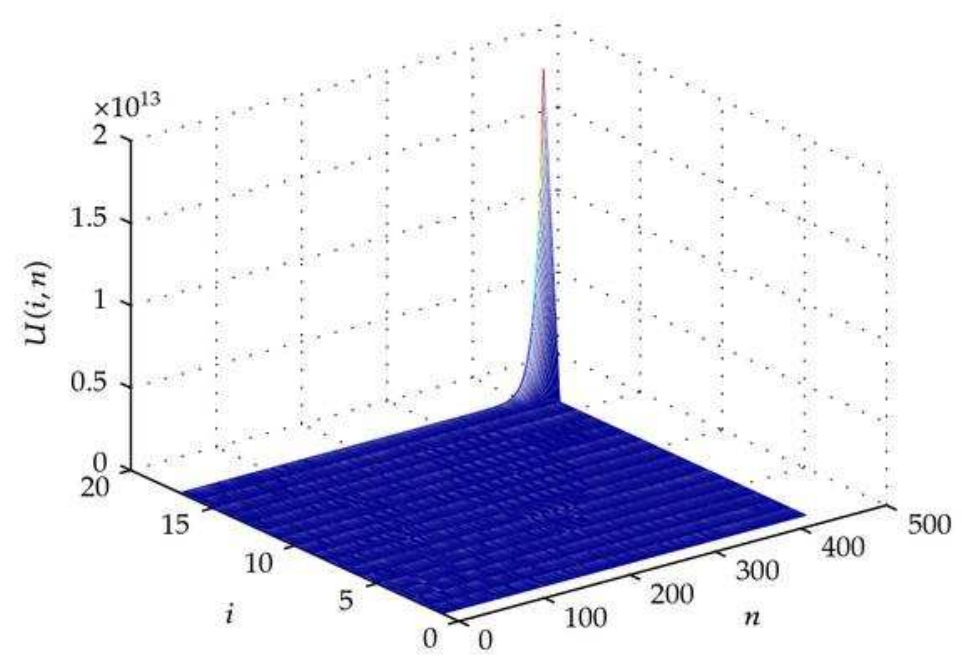

Figure 2: 2(a): Exact solution of the Cauchy Problem and Numerical Solution of system; 2(b): Numerical Solutions of systems $(a=1$ and $x *=1)$.

Considering further the relation $\mathrm{y}^{\prime} \mathrm{t}=\mathrm{tx}^{\prime} \mathrm{t}$, we transform (18) to the form

$$
y_{t}^{\prime}=\frac{t}{f(x, y, t)}
$$

Equations (18) and (19) represent a system of coupled first-order equations with respect to functions $\mathrm{x}=\mathrm{x}(\mathrm{t})$ and $y=y(t)$. The system (18)-(19) should be defined with the initial conditions

$$
x\left(t_{0}\right)=x_{0}, \quad y\left(t_{0}\right)=y_{0}, \quad t_{0}=y_{1}
$$

The Cauchy problem (18)-(20) can be integrated numerically applying the standard numerical methods [5, 6], without fear of blow-up solutions.

Remark.The systems of equations (9) and (16) are special cases of parametrically defined nonlinear differential equations, which are considered in $[9,10]$. In [10], general solutions of several parametrically determined ODEs were obtained using differential transformations based on the introduction of a new independent variable $\mathrm{t}=\mathrm{y}^{\prime} \mathrm{x}$.

\section{REFERENCES}

1. G. Acosta, G. Dur'an, J. D. Rossi, Computing, 2002, Vol. 68 343-373.

2. S. Moriguti, C. Okuno, R. Suekane, M. Iri, K. Takeuchi, IkiteiruSuugaku - SuuriKougaku no Hatten (in Japanese), Baifukan, Tokyo, 1979.

3. C. Hirota, K. Ozawa, J. Comp. Appl. Math. 193 (2) (2006) 614-637.

4. P. G. Dlamini, M. Khumalo, Math. Probl. Eng. 6 (2012) Art. ID 162034.

5. W. E. Schiesser. Computational Mathematics in Engineering and Applied Science: ODEs, DAEs, and PDEs. CRC Press, Boca Raton, 1994.

6. U. M. Ascher, L. R. Petzold, Computer Methods for Ordinary Differential Equations and Differential-Algebraic Equations, 
SIAM, 1998

7. A. D. Polyanin, V. F. Zaitsev, Handbook of Exact Solutions for Ordinary Differential Equations, Chapman \& Hall/CRC Press, Boca Raton, 2003.

8. N. A. Kudryashov. Analytical Theory of Nonlinear Differential Equations (in Russian). Institute of Computer Science, Moscow-Izhevsk, 2004.

9. A. D. Polyanin, A. I. Zhurov, Appl. Math. Lett. 55 (2016) 72-80.

10. A. D. Polyanin, A. I. Zhurov, Appl. Math. Lett. 64 (2017) 59-66.

11. X.-Y. Chen, H. Matano, M. Mimura, Finite-point extinction and continuity of interfaces in a nonlinear diffusion equation with strong absorption. J. ReineAngew. Math. 459 (1995), 1-36.

12. A. Constantin, J. Escher, Well-posedness, global existence, and blowup phenomena for a periodic quasi-linear hyperbolic equation. Comm. Pure Appl. Math. 51 (1998), 475-504.

13. J. W. Dold, Analysis of the early stage of thermal runaway. Quart. J. Mech. Appl. Math.38 (1985), 361-387.

14. J. W. Dold, V. A. Galaktionov, A. A. Lacey, J. L. V'azquez, Rate of approach to a singularsteady state in quasilinear reactiondiffusion equations. Ann. ScuolaNormale Super. Pisa 26(1998), 663-687.

15. S. Dimova, M. Kaschiev, M. Koleva, D. Vasileva, Numerical analysis of radially nonsymmetricblow-up solutions of a nonlinear parabolic problem. J. Comput. Appl. Math. 97 (1998),81-97.

16. W. E. and B. Engquist, Blowup of solutions of the unsteady Prandtl's equation. Comm. PureAppl. Math. 50 (1997), 12871293.

17. C. Elliott, Z. Songmu, On the Cahn-Hilliard equation. Arch. Rat. Mech. Anal. 96 (1986),339-357.

18. M. Escobedo, M. A. Herrero, Boundedness and blowup for a semilinear reaction-diffusionsystems. J. Differ. Equat. 89 (1991), $176-202$

19. Sivasakthi, A. (2018). An Investigation on Heavy Oil Viscosity Through Viscometer and Numerical Corrections. International Journal of Mechanical and Production Engineering Research and Development, 8(4).

20. Yaseen, N. D., Chiad, J. S., \& Ghani, F. M. A. (2018). The Study and Analysis of Stress Distribution Subjected on the Replacement Knee Joint Components using Photo-Elasticity and Numerical Methods. International Journal of Mechanical and Production Engineering Research and Development (IJMPERD), 8(06), 449-464.

21. Praveen, J., Pathan, M., \& Darvekar, S. K. Numerical Simulation Of Plug Valve Design Parameters As Per Standards.

22. Kumar, P. K., \& Subrahmanyam, J. Analytical And Numerical Approach To Vibration Analysis Of Liquid Filled Aluminum Thincylindrical Shells. 